\title{
Error Rates of a Full-duplex System over EGK Fading Channels Subject to Laplacian Interference
}

\author{
Hamza Soury, Member, IEEE, Hesham ElSawy, Member, IEEE, \\ and Mohamed-Slim Alouini, Fellow, IEEE \\ E-mail: \{soury.hamza, hesham.elsawy, slim.alouini\}@kaust.edu.sa.
}

\begin{abstract}
This paper develops a mathematical paradigm to study downlink error rates and throughput for half-duplex (HD) terminals served by a full-duplex (FD) base station (BS). Particularly, we study the dominant intra-cell interferer problem that appears between HD users scheduled on the same FDchannel. The distribution of the dominant interference is first characterized via its distribution function, which is derived in closed-form. Assuming Nakagami- $m$ fading, the probability of error for different modulation schemes is studied and a unified closed-form expression for the average symbol error rate is derived. To this end, we show the effective downlink throughput gain, harvested by employing FD communication at a BS that serves HD users, as a function of the signal-to-interference-ratio when compared to an idealized HD interference and noise free BS operation.
\end{abstract}

Index Terms-Dominant interference, Gaussian noise, Laplace interference, Nakagami- $m$ fading, error rate, throughput.

\section{INTRODUCTION}

In-band full-duplex (FD) communication is introduced as a promising technology to improve spectral utilization in cellular networks [1], [2]. FD communication relies on the recently developed self-interference (SI) cancellation techniques that enable simultaneous transmission and reception on the same frequency band, which offer better spectral utilization compared to the half-duplex (HD) system [3], [4]. However, implementing SI cancellation at the user's equipment (UE) can be expensive in terms of complexity, power consumption, and/or terminal price. Furthermore, cellular networks operators can only enforce FD upgrade at the base station (BS) side not the UE side.

The 3-node topology (3NT) is proposed to serve HD UEs by FD BSs and yet improves the spectral utilization [5]-[7]. In the 3NT, the BS simultaneously reuses the uplink channel assigned for one UE to serve another downlink UE and vice versa, as shown in Fig. 1. Compared to the HD case, the 3NT requires half the number of channels to serve the same number of users, and hence, the bandwidth (BW) occupied by each channel is doubled. Doubling the channel BW directly implies doubling the transmission rate, but not necessarily doubling the throughput. This is because the BW improvement offered by the $3 \mathrm{NT}$ comes at the expense of creating SI at the BS side and intra-cell interference at the UE side, which impose decoding errors. The BS exactly knows the interfering codeword and can accurately estimate the SI channel, and hence, SI can be sufficiently suppressed via SI cancellation techniques [1], [3],

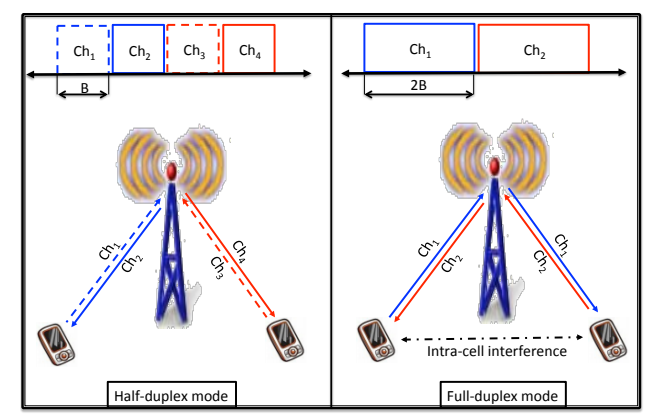

Fig. 1: Channel assignment in the 3NT FD and HD schemes.

[4]. However, the UE does not know the signal transmitted by the interfering UE, and hence, interference cancelation is not always viable.

This paper explicitly focuses on the intra-cell interference problem imposed by 3NT shown in Fig. 1. Therefore, we consider a single-cell scenario with FD BS and two HD UEs. Without loss of generality (WLOG), we assume that the spectrum can be either divided into four non-overlapping HD channels or two non-overlapping channels that are assigned using the 3NT FD mode, as shown in Fig. 1. In the HD case, each user is assigned two interference free $B \mathrm{~Hz}$ channels for uplink and downlink communication. On the other hand, the 3NT FD mode doubles the channels BW in both the uplink and downlink directions at the expense of SI in the uplink and intra-cell interference in the downlink. Many works dealt with SI cancellation at BS [8], [9]. In this work, we focus only on the downlink performance of one of the UEs while the performance of the uplink is out of the scope of this paper.

To eliminate the intra-cell interference problem in 3NT, the authors in [6] propose an interference alignment technique. The authors in [10] mitigate the intra-cell interference in 3NT via optimized channel and power allocation. However, the algorithms in [6], [10] require the knowledge of instantaneous mutual channel state information between interfering UEs, which is hard to estimate and/or communicate. A practical way to mitigate the intra-cell interference is to pair interfering UEs based on their statistical mutual channel conditions, which requires statistical studies for the intra-cell interference.

This paper presents a statistical study for intra-cell interference in 3NT FD cellular networks. Assuming Rayleigh fading 
on the mutual channel between the interfering UEs and that the interfering UE is transmitting Gaussian signals ${ }^{1}$, we show that the intra-cell interference has circularly symmetric Laplacian distribution with dependent in-phase and quadrature components. Then, downlink error rates for binary phase shift keying (BPSK), pulse amplitude modulation (PAM), and quadrature amplitude modulation (QAM) modulations in unfaded, and Nakagami- $m$ faded downlink channel are characterized. To this end, a unified closed-from expression for the average symbol error rate (SER) is derived and the throughput of the 3NT FD scheme is characterized and compared to its HD counterpart. It is worth mentioning that the Nakagami- $m$ fading model is selected for its flexibility to capture different fading environments and severities [13]. To the best of the authors' knowledge, this paper is the first to characterize the intra-cell interference in $3 \mathrm{NT}$ in closed-form and present the associated error rate and throughput analysis.

In what follows, subscripts $r$ and $i$ denote the real and imaginary parts, respectively, and $\rho(x)=1-\frac{1}{x}$.

\section{SySTEM MODEL}

According to proposed system described above, the complex base-band received signal at the test UE can be expressed as

$$
r=\sqrt{P_{d} \mathcal{A}_{d}} h s+\sqrt{P_{u} \mathcal{A}_{u}} g x+n,
$$

where $P_{d}$ is the downlink transmit power, $\mathcal{A}_{d}$ is a constant that captures downlink large-scale power attenuation, $s$ is the intended symbol drawn from a unit power constellation, $h$ is the intended channel with unit-mean Nakagami- $m$ distributed power gain, $P_{u}$ is the power transmitted by the interfering UE, $\mathcal{A}_{u}$ is a constant that captures large-scale interference power attenuation, $x$ is the intra-cell interfering Gaussian symbol, $g$ is the unit variance circularly symmetric complex Gaussian mutual channel gain between the UEs, and $n$ is the additive white noise. The random variables $h, s, g, x$, and $n$ are assumed to be independent.

Remark 1 The distribution of $g$ models a severe Rayleigh fading scenario, which occurs in non-line of sight (NLOS) scattering rich environments. The Rayleigh fading assumption on the interference channel can be justified by the ability of the BS to exploit multi-user diversity to reuse channels between UEs that have NLOS interfering link.

Remark 2 Unlike $g$, which is assumed Rayleigh by virtue of the multi-user diversity, $h$ is modeled using the more general Nakagami- $m$ fading distribution which models diverse types of fading channels that can appear between the user and the BS.

Remark 3 Gaussian signals provide accurate approximation for the interference generated from a single node transmitting on a faded channel [11]. This assumption is also validated in Section V of this paper.

\footnotetext{
${ }^{1}$ The Gaussian signaling abstraction for the interfering symbols is shown to have negligible effect on the error rate performance [11], [12]. The Gaussian signaling assumption is also validated in this paper.
}

For tractability, we assume dominant intra-cell interference and ignore the noise effect. The accuracy of this approximation is validated in Section V with realistic noise power. The approximated received signal without the noise effect is

$$
r \approx \sqrt{P_{d} \mathcal{A}_{d}} h s+\sqrt{P_{u} \mathcal{A}_{u}} g x .
$$

The received signal $r$ is a complex valued random variable with real and imaginary components, denoted as $r=\mathfrak{R}(r)+$ $j \mathfrak{I}(r)$, where $\mathfrak{R}$ and $\mathfrak{I}$ denote the real and imaginary parts respectively, and $j^{2}=-1$. To study (2), we first focus on the interference term, which can be considered as an additive complex perturbation for the useful symbol $s$. In the next section, we study the normalized intra-cell interference, denoted as $Z=g x$.

\section{Distribution OF THE INTRA-CELL INTERFERENCE}

Since $g$ and $x$ are both zero-mean unit-variance complex Gaussian random variables, their real and imaginary parts are independent Gaussian random variables with zero-mean and variance $\frac{1}{2}$. The intra-cell interference $Z$ can be expressed as

$$
Z=(\mathfrak{R}(g) \mathfrak{R}(x)-\mathfrak{I}(g) \mathfrak{I}(x))+j(\mathfrak{R}(g) \mathfrak{I}(x)+\mathfrak{I}(g) \mathfrak{R}(x)) .
$$

Lemma 1 gives the characteristic function (CHF) of $Z$, denoted by $\varphi_{Z}(u, v)$.

Lemma 1 The intra-cell interference from a dominant UE transmitting Gaussian signals over a Rayleigh faded channel has the following characteristic function

$$
\varphi_{Z}(u, v)=\frac{4}{4+u^{2}+v^{2}} .
$$

Proof: See AppendixA.

Remark 4 The expression in (4) is a special case of the bivariate Laplace distribution [14]. Furthermore, the CHF of $Z$ is independent of the angle between the real and imaginary components. Consequently, (4) shows that $Z$ follows a circularly symmetric complex Laplace distribution.

Theorem 1. The intra-cell interference from a dominant $U E$ transmitting Gaussian signals over a Rayleigh faded channel has the following cumulative distribution function (CDF)

$$
\begin{aligned}
& F_{Z}\left(z_{r}, z_{i}\right)=1-\frac{1}{2}\left(e^{-2 z_{r}}+e^{-2 z_{i}}\right) \\
& +\frac{1}{4 \pi} \mathrm{G}_{1,0,2,1,2,1}^{0,1,0,2,0,2}\left[\frac{1}{z_{r}^{2}}, \frac{1}{z_{i}^{2}} \mid \begin{array}{c|c|c}
0 & \frac{1}{2}, 1 & \frac{1}{2}, 1 \\
& 0 & 0
\end{array}\right],
\end{aligned}
$$

where G. $\because[.,[\cdot, \cdot]$ is the bivariate Meijer's $G$ function (BMGF), an extension of the Meijer's G function (MGF), that is defined in [15].

Proof: See AppendixB.

Corollary 1.1 The intra-cell interference from a dominant UE transmitting Gaussian signals over a Rayleigh faded channel has the following probability density function (PDF)

$$
f_{Z}\left(z_{r}, z_{i}\right)=\frac{2}{\pi} K_{0}\left(2 \sqrt{z_{r}^{2}+z_{i}^{2}}\right),
$$


where $K_{0}(\cdot)$ is the 0 -th order modified Bessel function of the second kind [16, Eq. (9.6.21)].

Proof: The PDF in (6) is obtained from the derivative of the CDF from Theorem 1. In fact, using the definition of the BMGF [15], the derivative of (5) is done with respect to $z_{r}$ and $z_{i}$. A double integral appears, which can be solved using the identity in [17, Eq. (2.9.5)], to get an expression of the PDF in terms of the MGF. Such special formula can be expressed in terms of the modified Bessel function of the second order as shown in [17, Eq. (2.9.31) \& Eq. (2.9.39)].

Remark 5 The PDF in (6) cannot be expressed as a product of the two marginal PDFs of $z_{r}$ and $z_{i}$, which proves the dependence among the real and imaginary components of $Z$.

Remark 6 The problem of decoding in the presence of additive Laplacian perturbation (i.e., noise or interference) has been widely addressed in the literature (see [13], [18] and the references therein). However, a commonly used assumption is that the real and imaginary components, of the Laplacian perturbation, are independent. Hence, the presented work contributes to the literature by modeling the case with dependent real and imaginary components. Furthermore, the Laplacian perturbation is rigorously derived in this paper for a system model not presented as an assumption.

\section{ERROR RATES \& THROUGHPUT}

This section studies downlink decoding errors that occur due to Laplacian intra-cell interference. In particular, the error rates of BPSK, PAM, and QAM are obtained. At first, conditional expressions (i.e., by conditioning on the downlink channel gain) are obtained in terms of the instantaneous signal-tointerference ratio (SIR), denoted as $\gamma=\frac{P_{d} \mathcal{A}_{d}|h|^{2}}{P_{u} \mathcal{A}_{u}}$. Then, a unified expression for the average SER is derived by averaging over the Nakagami- $m$ distribution of the downlink channel gain.

\section{A. Conditional Error Rate Analysis}

The conditional error rate analysis can also be considered as the error rate of unfaded downlink channel. Two types of modulations are considered, (i) one-dimensional constellation (1D), i.e. BPSK and M-PAM, and (ii) two-dimensional constellation (2D), i.e. M-QAM.

1) Error rate for $1 D$ constellations: The conditional error rates for the BPSK, PAM modulations are given in the following Theorem and Corollary.

Theorem 2. The conditional downlink bit error rate in $3 N T$ with Laplacian intra-cell interference and maximum likelihood detector for the BPSK modulation is given by

$$
P_{B P S K}(e \mid \gamma)=\frac{1}{2} e^{-2 \sqrt{\gamma}}
$$

Proof: Since the BPSK transmits real symbols $s= \pm E_{s}$, the conditional PDF of the received signal $r$ is obtained from (6) as $f_{r}(r)=e^{-2\left|r \mp E_{s}\right|}$. Given that the BPSK symbols are equiprobable, the probability of error can be computed as
$\operatorname{Pr}(e \mid \gamma)=\int_{0}^{\infty} f_{r}\left(r \mid S=-E_{s}\right) d r$. Then, (7) is obtained by setting $E_{s}=1$ due to the assumed unit energy constellation.

Corollary 2.1 The conditional downlink SER in 3NT with Laplacian intra-cell interference and maximum likelihood detector for the PAM modulation is given by

$$
P_{P A M}(e \mid \gamma)=\rho(M) e^{-2 \sqrt{\frac{3 \gamma}{M^{2}-1}}}
$$

Proof: The probability of error in PAM modulation can be derived directly from BPSK case as $P_{P A M}(e)=$ $2\left(1-\frac{1}{M}\right) P_{B P S K}(e)$. Hence, the conditional SER of PAM modulation can be deduced from (7).

2) Error rate for $2 D$ constellations: A rectangular $M$-QAM is formed by an $M_{I}$-PAM and $M_{Q}$-PAM, which means that $M=M_{I} M_{Q}$. Thus, the SER of rectangular QAM modulation is given in the following theorem.

Theorem 3. The conditional downlink bit error rate in $3 N T$ with Laplacian intra-cell interference and maximum likelihood detector for the rectangular QAM modulation is given by

$$
\begin{aligned}
& P_{Q A M}(e \mid \gamma)=\rho\left(M_{I}\right) e^{-2 \frac{\sqrt{3 \gamma}}{d}}+\rho\left(M_{Q}\right) e^{-2 \tau \frac{\sqrt{3 \gamma}}{d}} \\
& \quad-\frac{1}{\pi} \rho\left(M_{I}\right) \rho\left(M_{Q}\right) \mathrm{G}_{1,0,2,1,2,1}^{0,1,0,2,0,2}\left[\frac{d^{2}}{3 \gamma}, \frac{d^{2}}{3 \tau^{2} \gamma} \mid \begin{array}{c|c|c}
0 & \frac{1}{2}, 1 & \frac{1}{2}, 1 \\
0 & 0
\end{array}\right],
\end{aligned}
$$

where $d=\sqrt{\left(M_{I}^{2}-1\right)+\tau^{2}\left(M_{Q}^{2}-1\right)}$, and $\tau$ is the inphase-to-quadrature decision distance ratio defined as $\tau=\frac{d_{Q}}{d_{I}}$. The terms $d_{I}$ and $d_{Q}$ represent the in-phase and quadrature phase decision distance, respectively.

Proof: See AppendixC.

A special case of that result is the square QAM, where $M_{I}=$ $M_{Q}=\sqrt{M}$ and $\tau=1$.

The error rate for rectangular QAM modulation given in Theorem 3 is expressed in terms of the BMGF, which imposes high computational complexity. Therefore, we propose the following approximation.

Proposition 1 The conditional downlink bit error rate in 3NT with Laplacian intra-cell interference for the rectangular QAM modulation can be approximated by

$$
\begin{aligned}
P_{Q A M}^{A}(e \mid \gamma) & =\rho\left(M_{I}\right) e^{-2 \frac{\sqrt{3 \gamma}}{d}}+\rho\left(M_{Q}\right) e^{-2 \tau \frac{\sqrt{3 \gamma}}{d}} \\
& -\rho\left(M_{I}\right) \rho\left(M_{Q}\right) e^{-2(1+\tau) \frac{\sqrt{3 \gamma}}{d}}
\end{aligned}
$$

In addition, the SER of square QAM can be approximated also as

$$
P_{S Q A M}^{A}(e \mid \gamma)=2 \rho(\sqrt{M}) e^{-\sqrt{\frac{6 \gamma}{M-1}}}-\rho(\sqrt{M})^{2} e^{-2 \sqrt{\frac{6 \gamma}{M-1}}} .
$$

Proof: The approximation is obtained by considering a QAM modulation formed by two independent PAMs, namely, the in-phase and the quadrature phase. Thereby, a correct detection of the $M$-QAM appears only for a correct detection 
TABLE I: SER Modulation-Specific Parameters $n, a_{k}$, and $b_{k}$

\begin{tabular}{|c|c|c|c|c|}
\hline \multirow{2}{*}{$\begin{array}{c}\text { Modulation } \\
\text { Scheme }\end{array}$} & \multicolumn{5}{|c|}{ Modulation Specific Parameters } \\
\cline { 2 - 5 } & $n$ & $k$ & $a_{k}$ & $b_{k}$ \\
\hline BPSK & 1 & 1 & $\frac{1}{2}$ & 2 \\
\hline PAM & 1 & 1 & $\rho(M)$ & $2 \sqrt{\frac{3}{M^{2}-1}}$ \\
\hline Approximated & 2 & 1 & $2 \rho(\sqrt{M})$ & $\sqrt{\frac{6}{M-1}}$ \\
\cline { 3 - 5 } Square-QAM & & 2 & $-\rho(\sqrt{M})^{2}$ & $2 \sqrt{\frac{6}{M-1}}$ \\
\hline \multirow{2}{*}{$\begin{array}{c}\text { Approximated } \\
\text { Rectangular-QAM }\end{array}$} & \multirow{3}{*}{3} & 1 & $\rho\left(M_{I}\right)$ & $2 \frac{\sqrt{3}}{d}$ \\
\cline { 2 - 5 } & & 2 & $\rho\left(M_{Q}\right)$ & $2 \tau \frac{\sqrt{3}}{d}$ \\
\cline { 3 - 5 } & & $-\rho\left(M_{I}\right) \rho\left(M_{Q}\right)$ & $2(1+\tau) \frac{\sqrt{3}}{d}$ \\
\hline
\end{tabular}

in the $M_{I}$-PAM and $M_{Q}$-PAM. Consequently, the probability of error of $M$-QAM may be approximated as

$$
P_{Q A M}^{A}(e)=1-\left(1-P_{P A M_{I}}(e)\right)\left(1-P_{P A M_{Q}}(e)\right) .
$$

Then (10) is obtained by replacing (8) in (12).

Exploiting the QAM approximation in Proposition 1, unified error rate expression, for the considered modulation schemes, is given in the following proposition.

Proposition 2 The conditional downlink error rate in 3NT with Laplacian intra-cell interference and maximum likelihood detector for the rectangular BPSK, PAM, and approximated QAM modulation schemes is given by

$$
S E R=\sum_{k=1}^{n} a_{k} e^{-b_{k} \sqrt{\gamma}},
$$

where $n, a_{k}$, and $b_{k}$ are related to the used modulation as shown in Table I.

Proof: The result directly follow from Theorem 2, Corollary 2.1, and Equations (10) and (11).

\section{B. Average SER over Nakagami-m Fading Channel}

The PDF of the Nakagami- $m$ distribution is in essence a central chi-square PDF [19] given by

$$
p_{|h|}(|h|)=\frac{2 m^{m}}{\Omega^{m} \Gamma(m)}|h|^{2 m-1} \exp \left(-\frac{m|h|^{2}}{\Omega}\right)
$$

where $\Omega=\mathbb{E}\left[|h|^{2}\right]$, and $\frac{1}{2} \leq m$ is the fading shape parameter. $\mathbb{E}[\cdot]$ represented the expected value a random variable. Furthermore, let $\mathbb{E}\left[|h|^{2}\right]=1$, then the average SIR is given by $\bar{\gamma}=\mathbb{E}[\gamma]=\frac{P_{d} \mathcal{A}_{d}}{P_{u} \mathcal{A}_{y}}$. Consequently, the downlink SIR, $\gamma$, is distributed according to a Gamma distribution given by

$$
p_{\gamma}(\gamma)=\frac{m^{m} \gamma^{m-1}}{\bar{\gamma}^{m} \Gamma(m)} \exp \left(-\frac{m \gamma}{\bar{\gamma}}\right) .
$$

Using the Gamma distribution in (15), the exact average SER of rectangular QAM is given in the following theorem.
Theorem 4. The average SER of rectangular QAM in $3 N T$ with Nakagami-m fading on the downlink, Laplacian intracell interference, and maximum likelihood detector is obtained by

$$
\begin{aligned}
& \overline{S E R}_{Q A M}=\rho\left(M_{I}\right) \frac{\Gamma\left(m+\frac{1}{2}\right)}{\sqrt{\pi}} U\left(m, \frac{1}{2}, \frac{3 \bar{\gamma}}{m d^{2}}\right) \\
& +\rho\left(M_{Q}\right) \frac{\Gamma\left(m+\frac{1}{2}\right)}{\sqrt{\pi}} U\left(m, \frac{1}{2}, \frac{3 \tau^{2} \bar{\gamma}}{m d^{2}}\right) \\
& -\frac{\rho\left(M_{I}\right) \rho\left(M_{Q}\right)}{\pi \Gamma(m)} \mathrm{G}_{2,1,1,2,1,1}^{0,2,2,1,1}\left[\tau^{2}, \frac{m d^{2}}{3 \bar{\gamma}} \mid \begin{array}{c|c|c|c}
\frac{1}{2}, 1 & 1 & 0 \\
0 & \frac{1}{2}, 0 & m
\end{array}\right],
\end{aligned}
$$

where $U(\cdot, \cdot, \cdot)$ is the Tricomi hypergeometric function, defined in [16, Chapter 13].

Proof: From (9), the average SER can be determined by solving an integral in the form $\int_{0}^{\infty} p_{\gamma}(\gamma) P_{Q A M}(e \mid \gamma) d \gamma$. The first two terms can be solved using the identity [20, Eq. (6.2)] and expressed in terms of the Tricomi function thanks to [20, Eq. (6.44)]. The last term is solved using the Mellin representation of the BMGF [21].

Similar to Theorem 3, the results in Theorem 4 involve a BMGF that imposes high computational complexity. Therefore, a simple unified expression for the average SER of BPSK, PAM, and QAM (approximation), is given in the following theorem.

Theorem 5. The average SER for BPSK, PAM, and approximated QAM in $3 N T$ with Nakagami-m fading is given by

$$
\overline{S E R}=\sum_{k=1}^{n} \frac{a_{k}}{\sqrt{\pi}} \Gamma\left(m+\frac{1}{2}\right) U\left(m, \frac{1}{2}, \frac{b_{k}^{2} \bar{\gamma}}{4 m}\right) .
$$

where $a_{k}$ and $b_{k}$ are defined in Table I.

Proof: From (13), the average SER can be determined by solving the integral in the form $\int_{0}^{\infty} p_{\gamma}(\gamma) e^{-b_{k} \sqrt{\gamma}}$.

\section{Average SER over Rayleigh Fading Channel}

The Rayleigh distribution appears as a special case of the Nakagami- $m$ distribution by setting $m=1$, which is an interesting special case as it leads to simpler error rate expression. In such case, the exact average SER of rectangular QAM can be obtained with the help of [16, Chap. 13] as

$$
\begin{aligned}
& \overline{S E R}_{Q A M}=\rho\left(M_{I}\right)+\rho\left(M_{Q}\right)-\frac{2 \sqrt{3 \pi \gamma}}{d} \\
& \left(\rho\left(M_{I}\right) e^{\frac{3 \bar{\gamma}}{d^{2}}} Q(\sqrt{6 \bar{\gamma}} / d)+\rho\left(M_{Q}\right) \tau e^{\frac{3 \tau^{2} \bar{\gamma}}{d^{2}}} Q(\tau \sqrt{6 \bar{\gamma}} / d)\right) \\
& -\frac{1}{\pi} \rho\left(M_{I}\right) \rho\left(M_{Q}\right) \mathrm{G}_{2,1,1,2,1,1}^{0,2,2,1,1}\left[\tau^{2}, \frac{d^{2}}{3 \gamma} \mid \begin{array}{c|c|c}
\frac{1}{2}, 1 & 1 & 0 \\
0 & \frac{1}{2}, 0 & 1
\end{array}\right]
\end{aligned}
$$

In addition, the average SER for BPSK, PAM, and approximated QAM can be expressed in its simplest form as

$$
\overline{S E R}=\sum_{k=1}^{n} a_{k}\left(1-b_{k} \sqrt{\pi \bar{\gamma}} e^{\frac{b_{k}^{2} \bar{\gamma}}{4}} Q\left(b_{k} \sqrt{\bar{\gamma} / 2}\right)\right),
$$

where $Q(\cdot)$ is the Gaussian Q function [22, Eq. (A.1)]. 


\section{Throughput Analysis}

The error rate analysis shows the negative impact of the 3NT FD communication due to the imposed intra-cell interference. Looking into the throughput, the positive and negative impacts of 3NT are both captured in the improved BW and degraded SIR, respectively. The throughput is defined as

$$
\mathcal{T}=\frac{\log _{2}(M)(1-\overline{S E R})}{t_{s}},
$$

where $\log _{2}(M)$ is the number of bits per symbol, $t_{s}$ is the symbol duration. Using (20), the throughput gain imposed by the 3NT FD communication when compared to an idealized HD scenario (i.e. error free HD communication) is

$$
\mathcal{G}=\frac{2 B \log _{2}(M)(1-\overline{S E R})}{B \log _{2}(M)}=2(1-\overline{S E R}),
$$

where $\overline{S E R}$ is given in Theorem 5 and Theorem 4 .

\section{NUMERICAL RESULTS}

This section shows some selected numerical results supported by Monte Carlo Matlab ${ }^{\circledR}$ simulations to validate the developed mathematical paradigm and obtain insights into the 3NT FD operation. Particularly, we validate the Gaussian signaling assumption for the interfering symbol in (1), the dominance of the intra-cell interference when compared to the noise in (2), and the QAM error rate approximation in Proposition 1.

All results are plotted against the average SIR $\bar{\gamma}=\frac{P_{d} \mathcal{A}_{d}}{P_{u} \mathcal{A}_{u}}$, which is varied from $0 \mathrm{~dB}$ to $50 \mathrm{~dB}$ to capture the $3 \mathrm{NT}$ operation in different types of BSs. That is, we can infer the cell size (i.e., BS type) from the average SIR value. For instance, the transmit power of small BSs (e.g., femto and pico BSs) is comparable to the UE power, which is typically in the range of $200 \mathrm{~mW}$ to $1 \mathrm{~W}$. Therefore, in small BS scenario the average SIR term depends on the relative large-scale attenuation factors $\mathcal{A}_{d}$ and $\mathcal{A}_{u}$, which may lead to small values of $\bar{\gamma}$. Macro BSs transmit power is typically several tens of watts, and hence, in a Macro BSs scenario the downlink power $P_{d}$ dominates the average SIR term leading to high values of $\bar{\gamma}$.

In Fig. 2 the analysis is validated via two simulation scenarios, namely the "Noisy Simulation" and "Simulation Scenario 2". In the Noisy Simulation, the received signal is perturbed by a Gaussian noise in addition to the Laplacian interference, which perfectly matches the analysis and validates the derived SER expressions. The interfering UE in Simulation Scenario 2 randomly and uniformly alternates between BPSK and MQAM for $M \in\{4,16,64\}$. Hence, Simulation Scenario 2 validates the Gaussian signaling assumption of the interfering UEs. The figure shows that the accuracy of the Gaussian signaling assumption is better for faded downlink scenarios. This is because fading on the downlink increases the level of uncertainties in the detection process at the test UE, which put less significance on the interfering symbol distribution from the interfering UE and vice versa. Nevertheless, the Gaussian

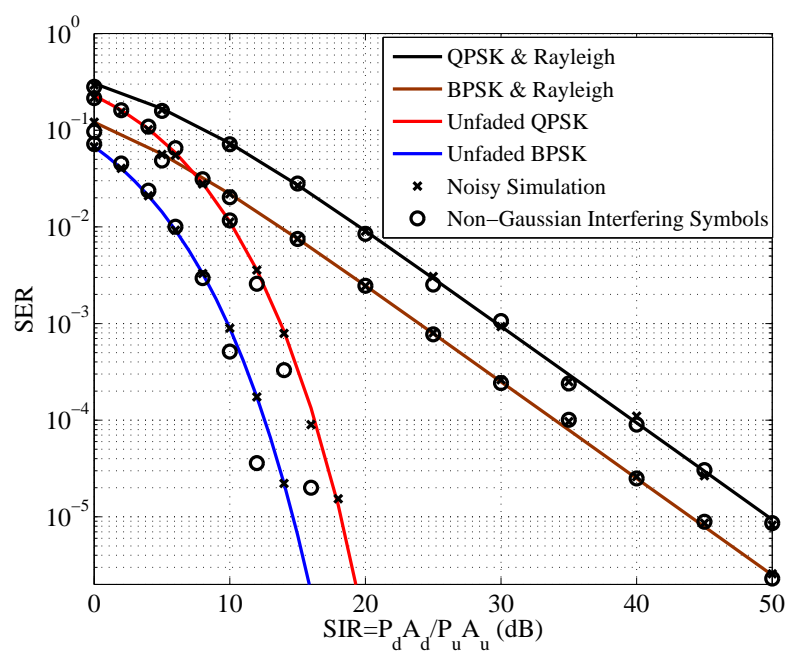

Fig. 2: SER of BPSK and QPSK schemes with and without Rayleigh fading.

signaling approximation always shows good accuracy in the low SIR regime, which is a critical region of operation to avoid throughput degradation.

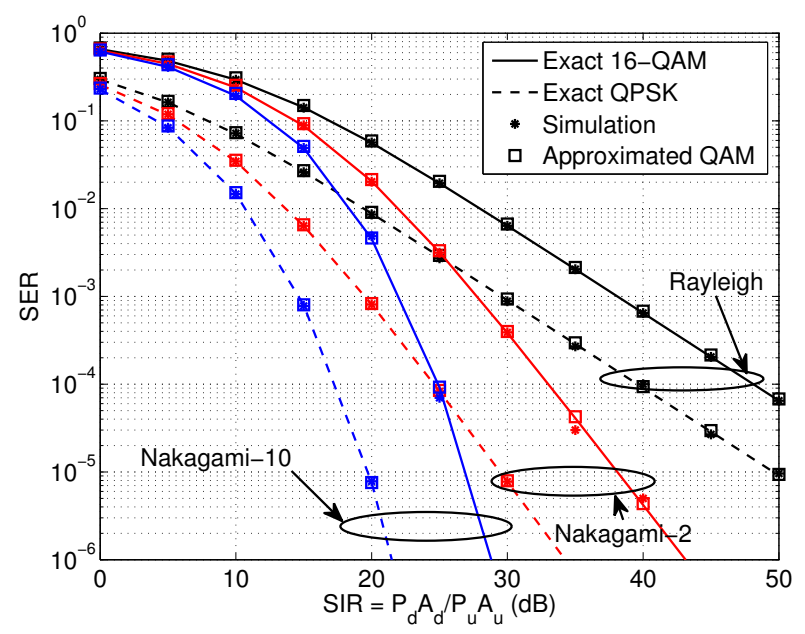

Fig. 3: SER of QPSK and 16-QAM schemes over Nakagami$m$ fading for $m=1,2$, and 10 and negligible noise. The stars denote the simulation results and the squares denote the approximation SER.

Fig. 3 shows the cost of intra-cell interference in terms of error probability for different modulation schemes and fading models on the downlink. Generally, the figure shows that intra-cell interference can severely degrade the downlink performance at low SIR, which may happen in small-cell scenario due to the small transmit power as well as the small BS footprint (i.e., users pairs can be close to each other). On the other hand, the effect of intra-cell interference diminishes 
in macro cells (i.e., at high $\bar{\gamma}$ value) specially for good downlink channel condition. The figure also shows that the gap between the constellations decreases by increasing the fading severity (from $5 \mathrm{~dB}$ to $2 \mathrm{~dB}$ for $\mathrm{SER}=10^{-5}$ ), which is due to the dominance of the fading conditions on the error probability.

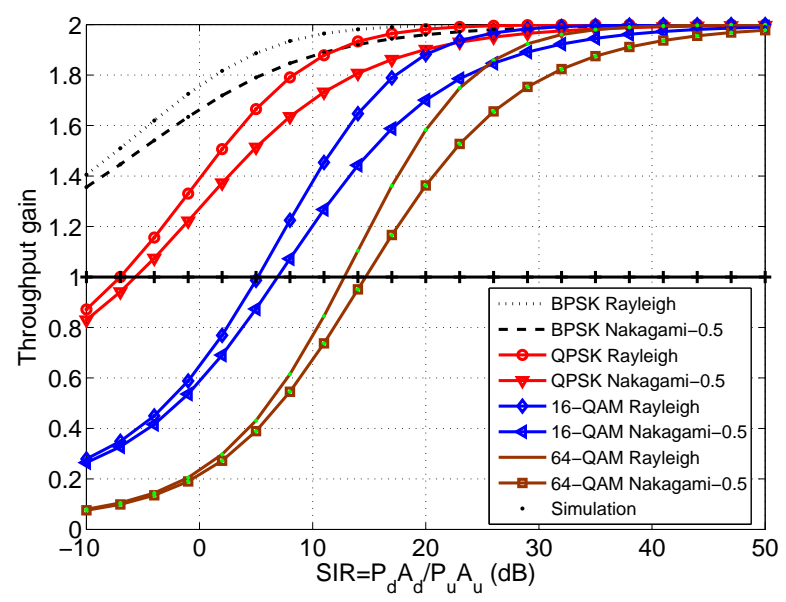

Fig. 4: Throughput gain of BPSK and QPSK over Rayleigh and Nakagami-0.5.

Fig. 4 shows the complete picture of the intra-cell interference effect imposed by the 3NT FD by looking at the throughput gain with respect to the interference free HD scenario. Interestingly, the figure shows that the throughput gain is always greater than unity for BPSK regardless of the SIR severity. This is because the FD doubles the transmission rate while the worst-case error probability for BPSK is one half. However, for higher modulation schemes, which are typical for downlink transmission, the intra-cell interference can significantly degrade the effective throughout. For instate, and $80 \%$ throughout degradation occurs for 64-QAM modulation at $0 \mathrm{~dB}$ SIR. On the other hand, at high SIR regime, 3NT doubles the effective throughput. The throughput gain, which relay on the developed error rate model, can be used to maximize the transmission rate via adaptive modulation based on the experienced SIR and fading severity.

\section{CONCLUSION}

This paper develops a mathematical paradigm to study downlink error rate and throughput for HD users when served by FD BSs via the 3-node topology. The paper derives closed form expressions for the intra-cell interference distribution as well as the SER for BPSK, PAM, and QAM modulation schemes in Nakagami- $m$ downlink channel fading. Finally, the paper characterizes effective throughput gain imposed by $3 \mathrm{NT}$ FD communication. The results show the severe degradation that may happen when employing 3NT FD in small cells, especially for schemes with big constellation sizes. On the other hand, 3NT provide nontrivial gains when employed in macro cells which high downlink transmission power. The developed paradigm can be used to adaptively change the modulation scheme according the the SIR and downlink channel condition to maximize the downlink throughput.

\section{APPENDIX A PROOF OF LEMMA 1}

The CHF is defined as $\varphi_{Z}(u, v)=\mathbb{E}\left[e^{j(u \Re(Z)+v \mathfrak{I}(Z))}\right]$. Using (3), $\varphi_{Z}$ can be written as

$$
\varphi_{Z}(u, v)=\mathbb{E}\left[e^{j x_{r}\left(u g_{r}+v g_{i}\right)} e^{j x_{i}\left(v g_{r}-u g_{i}\right)}\right] .
$$

Conditioning on $g_{r}$ and $g_{i}, \varphi_{Z}$ is written as the product of the CHF of $x_{r}$ and $x_{i}$. Thereby, the CHF of $Z$ is evaluated as the mean of that product over $g_{r}$ and $g_{i}$. Recall that the CHF of $x_{r}$ and $x_{i}$ have the same expression $\varphi_{x}(t)=e^{-t^{2}}$ (zero mean Gaussian with variance $\frac{1}{2}$ ). Also $g_{r}$ and $g_{i}$ have the same PDF, so $\varphi_{Z}$ appears as the square of the integral of the CHF of $x$ over the PDF of normal distribution. More specifically $\varphi_{Z}$ is equal to $\varphi_{Z}(u, v)=\frac{1}{\pi}\left(\int_{\mathbb{R}} e^{-\frac{1}{4} h_{I r}^{2}\left(4+u^{2}+v^{2}\right)} d h_{I r}\right)^{2}$. By the end, (4) is obtained by solving that integral.

\section{APPENDIX B \\ PROOF OF THEOREM 1}

The CDF is equal to $F_{Z}\left(z_{r}, z_{i}\right)=\bar{F}_{Z}\left(z_{r}, z_{i}\right)+F_{Z_{r}}\left(z_{r}\right)+$ $F_{Z_{i}}\left(z_{i}\right)-1 . F_{Z_{r}}\left(z_{r}\right)$ and $F_{Z_{i}}\left(z_{i}\right)$ denote the marginal CDFs of $Z_{r}$ and $Z_{i}$ respectively, defined as

$$
\left\{\begin{array}{l}
F_{Z_{r}}\left(z_{r}\right)=1-\frac{1}{2} e^{-2 z_{r}} \\
F_{Z_{i}}\left(z_{i}\right)=1-\frac{1}{2} e^{-2 z_{i}}
\end{array}\right.
$$

On the other hand, $\bar{F}_{Z}\left(z_{r}, z_{i}\right)$ is the complementary $\mathrm{CDF}$, that is defined as the primitive of the inverse Laplace transform of the CHF that vanishes at $(\infty, \infty)$

$\bar{F}_{Z}\left(z_{r}, z_{i}\right)=\frac{1}{4 \pi^{2}} \iint_{z_{r}, z_{i}} \iint_{u, v} e^{j\left(z_{r} u+z_{i} v\right)} \varphi_{Z}(u, v) d u d v d z_{r} d z_{i}$,

Since $\varphi_{Z}$ is even, $\bar{F}_{Z}$ becomes a cosine transform. After integration with respect to $z_{r}$ and $z_{i}, \bar{F}_{Z}$ is expressed as a sine transform

$$
\bar{F}_{Z}\left(z_{r}, z_{i}\right)=\frac{4}{\pi^{2}} \int_{0}^{\infty} \int_{0}^{\infty} \frac{\sin \left(z_{r} u\right) \sin \left(z_{i} v\right)}{u v\left(4+u^{2}+v^{2}\right)} d u d v .
$$

Using an alternative expression of the sine function in terms of the MGF [17, Eq. (2.9.7)] and its Mellin-Barnes representation [17, Eq. (1.1.2)], the integration over $u$ and $v$ can be solved directly. Furthermore, (B.2) can be re-written as

$$
\begin{aligned}
& \bar{F}_{Z}\left(z_{r}, z_{i}\right)=\frac{1}{4 \pi} \frac{1}{(2 \pi j)^{2}} \int_{\mathcal{L}_{1}} \int_{\mathcal{L}_{2}} \Gamma(1+s+t) \Gamma(s) \Gamma(t) \\
& \times \frac{\Gamma\left(\frac{1}{2}+s\right) \Gamma\left(\frac{1}{2}+t\right)}{\Gamma(1+s) \Gamma(1+t)} z_{r}^{-2 s} z_{i}^{-2 t} d s d t \\
&=\frac{1}{4 \pi} \mathrm{G}_{1,0,2,1,2,1}^{0,1,0,2,0,2}\left[\frac{1}{z_{r}^{2}}, \frac{1}{z_{i}^{2}} \mid \begin{array}{c|c|c}
0 & \frac{1}{2}, 1 & \frac{1}{2}, 1 \\
0 & 0
\end{array}\right],
\end{aligned}
$$

where $\mathcal{L}_{1}$ and $\mathcal{L}_{2}$ are the domain of integration of $s$ and $t$. The BMGF is obtained from its definition in [15]. 


\section{APPENDIX C \\ PROOF OF THEOREM 3}

A rectangular QAM constellation is formed by $M=M_{I} M_{Q}$ symbols. These symbols can be classified as follows: 4 corner symbols, $2\left(M_{I}-2\right)$ edge symbols with decision distance $d_{I}$, $2\left(M_{Q}-2\right)$ edge symbols with decision distance $d_{Q}$, and $\left(M_{I}-\right.$ $2)\left(M_{Q}-2\right)$ inner symbols. The conditional SER is obtained by averaging the probability of error of each symbol. Let us begin with the corner symbols, where their probability of error is given by

$$
\begin{aligned}
P_{e}^{c o r} & =1-\int_{-\frac{d_{I}}{2}}^{\infty} \int_{-\frac{d_{Q}}{2}}^{\infty} f_{Z}(x, y) d x d y=1-F_{Z}\left(\frac{d_{I}}{2}, \frac{d_{Q}}{2}\right) \\
& =\frac{1}{2}\left(e^{-d_{I}}+e^{-d_{Q}}\right)-\bar{F}_{Z}\left(\frac{d_{I}}{2}, \frac{d_{Q}}{2}\right) .
\end{aligned}
$$

The calculation of the probability of error of the edge points is similar for the in-phase point and the quadrature-phase points, hence we are focusing on the in-phase point, which can be obtained as

$$
\begin{aligned}
P_{e}^{e d g, I} & =1-\int_{-\frac{d_{I}}{2}}^{\infty} \int_{-\frac{d_{Q}}{2}}^{\frac{d_{Q}}{2}} f_{Z}(x, y) d x d y \\
& =\frac{1}{2}\left(e^{-d_{I}}+2 e^{-d_{Q}}\right)-2 \bar{F}_{Z}\left(\frac{d_{I}}{2}, \frac{d_{Q}}{2}\right) .
\end{aligned}
$$

Per consequence, the probability of error detection of a quadrature phase edge symbol is given by

$$
\begin{aligned}
P_{e}^{e d g, Q} & =\frac{1}{2}\left(e^{-d_{Q}}+2 e^{-d_{I}}\right)-2 \bar{F}_{Z}\left(\frac{d_{Q}}{2}, \frac{d_{I}}{2}\right) \\
& =\frac{1}{2}\left(e^{-d_{Q}}+2 e^{-d_{I}}\right)-2 \bar{F}_{Z}\left(\frac{d_{I}}{2}, \frac{d_{Q}}{2}\right) .
\end{aligned}
$$

Finally, the probability of error detection of an inner symbol is obtained as

$$
\begin{aligned}
P_{e}^{i n n} & =1-\int_{-\frac{d_{I}}{2}}^{\frac{d_{I}}{2}} \int_{-\frac{d_{Q}}{2}}^{\frac{d_{Q}}{2}} f_{Z}(x, y) d x d y \\
& =e^{-d_{I}}+e^{-d_{Q}}-4 \bar{F}_{Z}\left(\frac{d_{I}}{2}, \frac{d_{Q}}{2}\right) .
\end{aligned}
$$

Hence, the SER of rectangular QAM is computed by averaging the previous error rates over the probability of symbol occurrence

$$
\begin{aligned}
& P_{Q A M}(e \mid \gamma)=\frac{1}{M_{I} M_{Q}}\left(4 P_{e}^{c o r}+2\left(M_{Q}-2\right) P_{e}^{e d g, Q}\right. \\
& \left.+2\left(M_{I}-2\right) P_{e}^{e d g, I}+\left(M_{Q}-2\right)\left(M_{I}-2\right) P_{e}^{i n n}\right)
\end{aligned}
$$

Now by replacing the decision distances by their values $d_{I}=$ $\frac{2 \sqrt{3 \gamma}}{d}, d_{Q}=\tau d_{I}$, and after some algebraic manipulation, the result in Theorem 3 is obtained easily.

\section{REFERENCES}

[1] S. Hong, J. Brand, J. Choi, M. Jain, J. Mehlman, S. Katti, and P. Levis, "Applications of self-interference cancellation in 5G and beyond," IEEE Commun. Mag., vol. 52, no. 2, pp. 114-121, Feb. 2014.

[2] M. Mohammadi, H. A. Suraweera, Y. Cao, I. Krikidis, and C. Tellambura, "Full-duplex radio for uplink/downlink wireless access with spatially random nodes," IEEE Trans. on Commun., vol. 63, no. 12, pp. 52505266, Dec 2015.
[3] A. Sabharwal, P. Schniter, D. Guo, D. Bliss, S. Rangarajan, and R. Wichman, "In-band full-duplex wireless: Challenges and opportunities," IEEE J. Select. Areas Commun, vol. 32, no. 9, pp. 1637-1652, Sep. 2014.

[4] J. I. Jain, Mayank Choi, T. Kim, D. Bharadia, S. Seth, K. Srinivasan, P. Levis, S. Katti, and P. Sinha, "Practical, real-time, full duplex wireless," in Proc. of the 17th Annu. Int. Conf. on Mob. Comp. and Netw. (MobiCom'2011), Las Vegas, NV, USA, Sep. 2011, pp. 301-312.

[5] K. M. Thilina, H. Tabassum, E. Hossain, and D. I. Kim, "Medium access control design for full duplex wireless systems: challenges and approaches," IEEE Communications Magazine, vol. 53, no. 5, pp. 112120, May 2015.

[6] K. Sundaresan, M. Khojastepour, E. Chai, and S. Rangarajan, "Fullduplex without strings: Enabling full-duplex with half-duplex clients," in Proc. of the 20th Annu. Int. Conf. on Mobile Computing and Networking (MobiCom'2014), Maui, Hawaii, USA, Sep. 2014.

[7] A. AlAmmouri, H. ElSawy, O. Amin, and M. S. Alouini, "In-band $\alpha$ duplex scheme for cellular networks: A stochastic geometry approach," IEEE Trans. on Wirel. Commun., vol. 15, no. 10, pp. 6797-6812, Oct 2016.

[8] H. Lee, D. Kim, S. Kim, J. Kim, C. Lee, and D. Hong, "Rotated precoder based self-interference cancellation in full-duplex communication," in Proc. of Int. Conf. on Elec., Inf. and Comm. (ICEIC'2014), Kota Kinabalu, Malaysia, Jan 2014, pp. 1-2.

[9] B. J. Kim and H. G. Ryu, "Self-interference cancellation using MachZehnder modulator for full-duplex communication," in Proc. of Int. Conf. on Inf. and Comm. Tech. Conv. (ICTC'2014), Busan, South Korea, Oct 2014, pp. 858-863.

[10] M. Feng, S. Mao, and T. Jiang, "Joint duplex mode selection, channel allocation, and power control for full-duplex cognitive femtocell networks," Dig. Commun. and Net., vol. 1, no. 1, pp. 30-44, 2015.

[11] A. Giorgetti and M. Chiani, "Influence of fading on the Gaussian approximation for BPSK and QPSK with asynchronous cochannel interference," IEEE Trans. on Wireless Commun., vol. 4, no. 2, pp. 384-389, March 2005.

[12] L. H. Afify, H. ElSawy, T. Y. Al-Naffouri, and M.-S. Alouini, "The influence of Gaussian signaling approximation on error performance in cellular networks," IEEE Communications Letters, vol. 19, no. 12, pp. 2202-2205, Dec 2015.

[13] H. Soury and M.-S. Alouini, "Symbol error rate of MPSK over EGK channels perturbed by a dominant additive Laplacian noise," IEEE Trans. on Commun., vol. 63, no. 7, pp. 2511-2523, Jul. 2015.

[14] T. Eltoft, T. Kim, and T.-W. Lee, "On the multivariate Laplace distribution," IEEE Sig. Proces. Lett., vol. 13, no. 5, pp. 300-303, May 2006.

[15] I. Ansari, S. Al-Ahmadi, F. Yilmaz, M.-S. Alouini, and H. Yanikomeroglu, "A new formula for the BER of binary modulations with dual-branch selection over generalized-K composite fading channels," IEEE Trans. Commun., vol. 59, no. 10, pp. 2654-2658, Oct. 2011.

[16] M. Abramowitz and I. A. Stegun, Handbook of Mathematical Functions with Formulas, Graphs, and Mathematical Tables, ser. National Bureau of Standards Applied Mathematics Series. New York: Dover Publications, 1964.

[17] A. Kilbas and M. Saigo, H-Transforms : Theory and Applications (Analytical Method and Special Function), 1st ed. CRC Press, 2004.

[18] S. Jiang and N. C. Beaulieu, "Precise BER computation for binary data detection in bandlimited white Laplace noise," IEEE Trans. Commun., vol. 59, no. 6, pp. 1570-1579, Jun. 2011.

[19] M. K. Simon and M.-S. Alouini, Digital Communication Over Fading Channels, 1st ed. New York: Wiley, 2005.

[20] M. A. Chaudhry and S. M. Zubair, On a Class of Incomplete Gamma Function with Applications. Boca Raton-London-Ney York-Washington, D.C.: Chapman \& Hall/CRC, 2002.

[21] P. Mittal and K. Gupta, "An integral involving generalized function of two variables," in Indian Acad. Sci., 1972, pp. 117-123.

[22] H. Soury, F. Yilmaz, and M.-S. Alouini, "Average bit error probability of binary coherent signaling over generalized fading channels subject to additive generalized Gaussian noise," IEEE Commun. Lett., vol. 16, no. 6, pp. 785-788, June 2012 